\title{
What Is a Qualitative Calculus? A General Framework
}

\author{
Gérard Ligozat ${ }^{1}$ and Jochen Renz ${ }^{2}$ \\ 1 LIMSI-CNRS, Université Paris-Sud, 91403 Orsay, France \\ 2 National ICT Australia ${ }^{\star}$, Knowledge Representation and Reasoning Group \\ UNSW Sydney, NSW 2052, Australia
}

\begin{abstract}
What is a qualitative calculus? Many qualitative spatial and temporal calculi arise from a set of JEPD (jointly exhaustive and pairwise disjoint) relations: a stock example is Allen's calculus, which is based on thirteen basic relations between intervals on the time line. This paper examines the construction of such a formalism from a general point of view, in order to make apparent the formal algebraic properties of all formalisms of that type. We show that the natural algebraic object governing this kind of calculus is a non-associative algebra (in the sense of Maddux), and that the notion of weak representation is the right notion for describing most basic properties. We discuss the ubiquity of weak representations in various guises, and argue that the fundamental notion of consistency itself can best be understood in terms of consistency of one weak representation with respect to another.
\end{abstract}

\section{Introduction}

What is a qualitative temporal or spatial calculus? And: why should we care? An obvious, if not quite satisfactory way of answering the first question would consist in listing some examples of fairly well-known examples: on the temporal side, Allen's interval calculus [1] is the most famous candidate; others are the point calculus [24], the pointand-interval calculus [7], generalized interval calculi [14], or the INDU calculus [20]; on the spatial side, there are Allen-like calculi, such as the directed interval calculus [22], the cardinal direction calculus [16], which is a particular case of the $n$-point calculi [4], the rectangle calculus [3], and more generally the $n$-block calculi [5], as well as calculi stemming from the RCC-like axiomatics, such as the RCC-5 and RCC- 8 calculi [21], and various kinds of calculi, such as the cyclic interval calculus [2], the star calculi [19], or the preference calculi [8].

Why should we care? A first reason is that, as becomes soon apparent after considering some of the examples, many calculi share common properties, and are used in analogous ways: Take for instance Allen's calculus. It makes use of a set of basic relations, and reasoning uses disjunctions of the basic relations (representing incomplete knowledge), also called (disjunctive) relations. A relation has a converse relation, and relations can be composed, giving rise to an algebraic structure called Allen's algebra (which is a relation algebra, in Tarski's sense [23]). In applications, the knowledge is represented by temporal networks, which are oriented graphs whose nodes stand for

\footnotetext{
* National ICT Australia is funded through the Australian Government's Backing Australia's Ability initiative, in part through the Australian Research Council.

C. Zhang, H.W. Guesgen, W.K. Yeap (Eds.): PRICAI 2004, LNAI 3157, pp. 53-64, 2004

(C) Springer-Verlag Berlin Heidelberg 2004
} 
intervals, and labels on the arcs which are relations. In this context, a basic problem is determining whether a given network is consistent (the problem is known to be NPcomplete, [24]). Finally, when a network is consistent, finding a qualitative instantiation of it amounts to refining the network to an atomic sub-network which is still consistent: and this can be checked at the algebraic level.

Thus, it makes sense to ask the question: to what extent do those properties extend to the other calculi we mentioned above? As first discussed in [17], it soon appears that some properties of Allen's calculus do not extend in general. Some disturbing facts:

- As remarked by $[9,17]$, the algebras of some calculi are not relation algebras in the sense of Tarski, but more general algebras called non-associative algebras by Maddux (relation algebras being the particular case of associative non-associative algebras). In fact, the INDU algebra is only a semi-associative algebra.

- The natural or intended models of the calculus may not be models in the strong sense or, in algebraic terms, representations of the algebra. This is no new realization: Allen's composition, for instance, expresses necessary and sufficient conditions only if the intervals are in a dense and unbounded linear ordering. But what is less known, apart from the fact that it may be interesting to reason in weaker structures, e.g., about intervals in a discrete linear ordering, is the fact that all such models correspond to weak representations of the algebra, in the sense of [13].

- For some calculi, such as the containment algebra [12] or the cyclic interval calculus [2], it has been observed that some finite atomic constraint networks which are algebraically closed $^{1}$ are not consistent. Again, this phenomenon is best expressed, if not explained, in terms of weak relations.

- For Allen's calculus, any consistent atomic network is in fact $k$-consistent, for all $k<n$, if it has $n$ nodes. Again, the analogous result is false for many calculi, and considering the various weak representations helps to explain why it may be so.

So we cannot hope to have general methods and have to look closer at what the calculi have to offer. Defining a family of calculi by giving examples amounts to a partial extensional definition. But what would an intensional definition be? If we can answer this last question, we have some hope of developing general methods which could be used for whole classes of calculi, instead of specific ones which have to be reinvented for each particular calculus.

Although we do not consider this particular aspect in this paper, an example of a general concept which is valid for a whole class of calculi is the notion of pre-convexity [15] which has been shown as providing a successful way of searching for tractable classes, at least for formalisms based on linear orderings such as Allen's calculus.

The purpose of this paper is to give a precise technical answer to the first question: what is a qualitative calculus? The answer involves a modest amount of - actually, two - algebraic notions, which both extend standard definitions in universal algebra: the notion of a non-associative algebra (which generalizes that of a relation algebra), and the notion of a weak representation, (which generalizes that of a representation).

\footnotetext{
${ }^{1}$ We use the term algebraically closed, or a-closed, to refer to the notion which is often (in some cases incorrectly) referred to as path-consistency: for any 3-tuple $(i, j, k)$ of nodes, composing the labels on $(i, k)$ and $(k, j)$ yields a result which contains the label on $(i, j)$.
} 
This paper provides a context for discussing these various points. In section 2 , the general construction of JEPD relations is presented in terms of partition schemes. The main operation in that context is weak composition, whose basic properties are discussed. Section 3 describes some typical examples of the construction. It is shown in Section 4 that all partition schemes give rise to non-associative algebras, and in Section 5 that the original partition schemes are in fact weak representations of the corresponding algebra. A proposal for a very general definition of a qualitative calculus is presented in Section 6 as well as a description of the various guises into which weak representations appear: both as particular kind of network and as natural universes of interpretation. Section 7 is concerned with the basic notion of consistency, which appears as a particular case of a more general notion of consistency of one weak representation with respect to another.

\section{Developing a New Calculus}

Although there seems to be almost no end to defining qualitative spatial or temporal calculi, most constructions are ultimately based on the use of a set of JEPD (jointly exhaustive and pairwise disjoint ${ }^{2}$ ) relations. This will be our starting point for defining a generic qualitative calculus, in a very general setting.

\subsection{Partition Schemes}

We start with a non-empty universe $U$, and consider a partition of $U \times U$ into a family of non-empty binary relations $\left(R_{i}\right)_{i \in I}$ :

$$
U \times U=\bigcup_{i \in I} R_{i}
$$

The relations $R_{i}$ are called basic relations. Usually, calculi defined in this way use a partition into a finite number of relations. In order to keep things simple, we assume $I$ to be a finite set. In concrete situations, $U$ is a set of temporal, spatial, or spatio-temporal entities (time points, intervals, regions, etc.). Among all possible binary relations, the partition selects a finite subset of "qualitative" relations which will be a basis for talking about particular situations. For instance, in Allen's calculus, $U$ is the set of all intervals in the rational line, and any configuration is described in terms of the 13 basic relations.

We make some rather weak assumptions about this setup. First, we assume that the diagonal (the identity relation) is one of the $R_{i} \mathrm{~s}$, say $R_{0}$ :

$$
R_{0}=\Delta=\{(u, v) \in U \times U \mid u=v\}
$$

Finally, we choose the partition in such a way that it is globally invariant under conversion. Recall that, for any binary relation $R, R \smile$ is defined by:

$$
R^{\smile}=\{(u, v) \in U \times U \mid(v, u) \in R\}
$$

\footnotetext{
${ }^{2}$ Contrary to one of the authors' initial assumption, the JEPD acronym does not seem to be related in any way to the JEPD hypothesis in biblical exegesis, where J, E, P, D stand for the Jehovist, Elohist, Priestly and Deuteronomist sources, respectively!
} 
We assume that the following holds:

$$
(\forall i \in I)(\exists j \in I) \quad R_{i}^{\smile}=R_{j}
$$

Definition 1. A partition scheme is a pair $\left(U,\left(R_{i}\right)_{i \in I}\right)$, where $U$ is a non-empty set and $\left(R_{i}\right)_{i \in I}$ a partition of $U \times U$ satisfying conditions (2) and (4).

\subsection{Describing Configurations}

Once we have decided on a partition scheme, we have a way of describing configurations in the universe $U$. Intuitively, a configuration is a (usually finite) subset $V \subseteq U$ of objects of $U$. By definition, given such a subset, each pair $(u, v) \in V \times V$ belongs to exactly one $R_{i}$ for a well-defined $i$. Later, we will think of $V$ as a set of nodes of a graph, and of the map $\nu: V \times V \rightarrow I$ as a labeling of the set of arcs of the graph. Clearly, $\nu(u, u)$ is the identity relation $R_{0}$, and $\nu(v, u)$ is the transpose of $\nu(u, v)$. The resulting graphs are called constraint networks in the literature. More generally, we can express constraints using Boolean expressions using the $R_{i}$ s. In particular, constraint networks using disjunctive labels are interpreted as conjunctions of disjunctive constraints represented by unions of basic relations on the labels.

\subsection{Weak Composition}

Up to now, we did not consider how constraints can be propagated. This is what we do now by defining the weak composition of two relations. Recall first the definition of the composition $R \circ S$ of two binary relations $R$ and $S$ :

$$
(R \circ S)=\{(u, v) \in U \times U \mid(\exists w \in U) \quad(u, w) \in R \quad \& \quad(w, v) \in S\}
$$

Weak composition, denoted by $R_{i} \diamond R_{j}$, of two relations $R_{i}$ and $R_{j}$ is defined as follows:

$$
\left(R_{i} \diamond R_{j}\right)=\bigcup_{k \in J} R_{k} \text { where } k \in J \text { if and only if }\left(R_{i} \circ R_{j}\right) \cap R_{k} \neq \emptyset
$$

Intuitively, weak composition is the best approximation we can get to the actual composition if we have to restrict ourselves to the language provided by the partition scheme. Notice that weak composition is only defined with respect to the partition, and not in an absolute sense, as is the case for the "real" composition.

At this level of generality, some unpleasant facts might be true. For instance, although all relations $R_{i}$ are non-empty by assumption, we have no guarantee that $R_{i} \diamond R_{j}$, or $R_{i} \circ R_{j}$ for that matter, are non-empty. A first remark is that weak composition is in a natural sense an upper approximation to composition:

Lemma 1. For any $i, j \in I: \quad R_{i} \diamond R_{j} \supseteq R_{i} \circ R_{j}$

Proof. Any $(u, v) \in R_{i} \circ R_{j}$ is in some (unique) $R_{k}$ for a well-defined $k$. Since this $R_{k}$ has an element in common with $R_{i} \circ R_{j}, R_{k}$ must belong to $R_{i} \diamond R_{j}$.

Lemma 2. For any $i, j, k \in I: \quad\left(R_{i} \diamond R_{j}\right) \cap R_{k}=\emptyset$ if and only if $\left(R_{i} \circ R_{j}\right) \cap R_{k}=\emptyset$ 
Proof. Because of Lemma 1, one direction is obvious. Conversely, if $\left(R_{i} \diamond R_{j}\right) \bigcap R_{k}$ is not empty, then, since $\left(R_{i} \diamond R_{j}\right)$ is a union of $R_{l} \mathrm{~s}, R_{k}$ is contained in it. Now, by definition of weak composition, this means that $R_{k}$ intersects $R_{i} \circ R j$.

The interaction of weak composition with conversion is an easy consequence of the corresponding result for composition:

Lemma 3. For all $i, j \in I: \quad\left(R_{i} \diamond R_{j}\right)^{\smile}=R_{j}^{\smile \diamond R_{i}^{\smile}}$

\subsection{Weak Composition and Seriality}

In many cases, the relations in the partition are serial relations. Recall that a relation $R$ is serial if the following condition holds:

$$
(\forall u \in U)(\exists v \in U) \text { such that }(u, v) \in R
$$

Lemma 4. If the relations $R$ and $S$ are serial, then $R \circ S$ is serial, (hence it is nonempty).

Proof. If $R$ and $S$ are serial, then, for an arbitrary $u$, choose first $w$ such that $(u, w) \in R$, then $v$ such that $(w, v) \in S$. Then $(u, v) \in(R \circ S)$.

As a consequence, since all basic relations are non-empty, the weak composition of two basic relations is itself non-empty.

Lemma 5. If the basic relations are serial, then $\forall i \in I: \quad \bigcup_{j \in I}\left(R_{i} \diamond R_{j}\right)=U \times U$

Proof. We have to show that, for any given $i$, and any pair $(u, v)$, there is a $j$ such that $(u, v)$ is in $R_{i} \diamond R_{j}$. We know that $(u, v) \in R_{k}$, for some well-defined $k$. Because $R_{i}$ and $R_{k}$ are serial, for all $t$ there are $x$ and $y$ such that $(t, x) \in R_{i}$ and $(t, y) \in R_{k}$. Therefore $(x, y) \in R_{i}^{\smile} \circ R_{k}$, so $R_{i}^{\smile} \circ R_{k}$ is non-empty. Moreover, there is one welldefined $j$ such that $(x, y) \in R_{j}$. Hence $(t, y)$ is both in $R_{k}$ and in $R_{i} \circ R_{j}$. Therefore, $R_{k} \subseteq\left(R_{i} \diamond R_{j}\right)$, hence $(u, v) \in\left(R_{i} \diamond R_{j}\right)$.

\section{Examples of Partition Schemes}

Example 1 (The linear ordering with two elements). Let $U=\{a, b\}$ a set with two elements. Let $R_{0}=\{(a, a),(b, b)\}, R_{1}=\{(a, b)\}, R_{2}=\{(b, a)\}$. The two-element set $U$, in other words, is linearly ordered by $R_{1}$ (or by $R_{2}$ ). Then $R_{1} \circ R_{1}=R_{2} \circ R_{2}=\emptyset$, $R_{1} \circ R_{2}=\{(a, a)\}$, and $R_{2} \circ R_{1}=\{(b, b)\}$. Hence $R_{1} \diamond R_{1}=\emptyset, R_{2} \diamond R_{2}=\emptyset$, $R_{1} \diamond R_{2}=R_{0}$, and $R_{2} \diamond R_{1}=R_{0}$.

Example 2 (The linear ordering with three elements). Let $U=\{a, b, c\}$ a set with three elements. Let $R_{0}=\{(a, a),(b, b),(c, c)\}, R_{1}=\{(a, b),(b, c),(a, c)\}, R_{2}=$ $\{(b, a),(c, b),(c, a)\}$. Here, the three-element set $U$ is linearly ordered by $R_{1}$ (or by $\left.R_{2}\right)$. Then $R_{1} \circ R_{1}=\{(a, c)\}, R_{2} \circ R_{2}=\{(c, a)\}, R_{1} \circ R_{2}=R_{2} \circ R_{1}=\{(a, a),(b, b)$, $(a, b),(b, a)\}$. Consequently, $R_{1} \diamond R_{1}=R_{1}, R_{2} \diamond R_{2}=R_{2}, R_{1} \diamond R_{2}=R_{2} \diamond R_{1}=U \times U$.

Example 3 (The point algebra). The standard example is the point algebra, where $U$ is the set $\mathrm{Q}$ of rational numbers, and $R_{1}$ is the usual ordering on $\mathrm{Q}$, denoted by $<. R_{2}$ is the converse of $R_{1}$. Because this ordering is dense and unbounded both on the left and on the right, we have $R_{1} \circ R_{1}=R_{1}, R_{2} \circ R_{2}=R_{2}, R_{2} \circ R_{1}=R_{1} \circ R_{2}=U \times U$. 
Example 4 (Allen's algebra). Here $U$ is the set of "intervals" in Q, i.e., of ordered pairs $\left(q_{1}, q_{2}\right) \in \mathbf{Q} \times \mathbf{Q}$ such that $q_{1}<q_{2}$. Basic relations are defined in the usual way [1]. Since $Q$ is dense and unbounded, weak composition coincides with composition [13].

Example 5 (Allen's calculus on integers). $U$ is the set of intervals in $Z$, that is, of pairs $\left(n_{1}, n_{2}\right) \in \mathbf{Z} \times \mathbf{Z}$ such that $n_{1}<n_{2}$. Weak composition differs from composition in this case: e.g., we still have $p \diamond p=p$, but the pair $([0,1],[2,3])$ is in $p$, but not in $p \circ p$.

\section{The Algebras of Qualitative Calculi}

\subsection{Algebras Derived from Partition Schemes}

Now we take an abstract algebraic point of view. For each $i \in I$, we introduce a symbol $r_{i}$ (which refers to $R_{i}$ ) and consider the set $B=\left\{r_{i} \mid i \in I\right\}$. Let $A$ be the Boolean algebra of all subsets of $B$. The top element of this algebra is denoted by 1 , and the bottom element (the empty set) by 0 . Union, intersection and complementation are denoted by,$+ \cdot$ and - , respectively. Let $\mathbf{1}^{\prime}$ denote $\left\{r_{0}\right\}$. We still denote by $r_{i}^{\smile}$ the operation of conversion. On this Boolean algebra, the weak composition function defines an operation which is usually denoted by ;. When tabulated, the corresponding table is called the weak composition table of the calculus. The operation of composition on basic symbols is extended to all subsets as follows:

$$
\text { For } a, b \in A,(a ; b)=\bigcup_{i, j}\left(r_{i} ; r_{j}\right), \text { where } r_{i} \in a \text { and } r_{j} \in b .
$$

Since the algebraic setup reflects facts about actual binary relations, the algebra we get in this way would be a relation algebra in Tarski's sense, if we considered composition. In the general case, however, what we are considering is only weak composition, an approximation to actual composition. What happens is that we get a weaker kind of algebra, namely, a non-associative algebra $[18,10]$ :

Definition 2. A non-associative algebra $A$ is a tuple $\mathcal{A}=\left(A,+,-, \mathbf{0}, \mathbf{1}, ;, \smile, \mathbf{1}^{\prime}\right)$ such that:

1. $(A,+,-, \mathbf{0}, \mathbf{1})$ is a Boolean algebra.

2. $\mathbf{1}^{\prime}$ is a constant, $\smile$ a unary and ; a binary operation s. t., for any $a, b, c \in A$ :
(a) $\left(a^{\smile}\right)^{\smile}=a$
(b) $\mathbf{1}^{\prime} ; a=a ; \mathbf{1}^{\prime}=a$
(c) $a ;(b+c)=a ; b+a ; c$

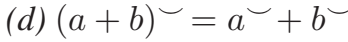
(e) $(a-b)^{\smile}=a^{\smile}-b^{\smile}$

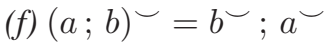
(g) $(a ; b) \cdot c^{\smile}=\mathbf{0}$ if and only if $(b ; c) \cdot a^{\smile}=\mathbf{0}$

A non-associative algebra is a relation algebra if it is associative.

Maddux [18] also introduced intermediate classes of non-associative algebras between relation algebras (RA) and general non-associative algebras (NA), namely weakly associative (WA) and semi-associative (SA) algebras. These classes form a hierarchy:

$$
\mathbf{N A} \supseteq \mathbf{W A} \supseteq \mathbf{S A} \supseteq \mathbf{R A}
$$

In particular, semi-associative algebras are those non-associative algebras which satisfy the following condition:

$$
\text { For all } a,(a ; \mathbf{1}) ; \mathbf{1}=a ; \mathbf{1} .
$$


Proposition 1. The algebraic structure associated to a partition scheme is a non-associative algebra. If the basic relations are serial, it is a semi-associative algebra.

Proof. We have to check points (2(a-g)) of Def.2 (checking the validity on basic relations is enough). The first six points are easily checked. The last axiom, the triangle axiom, holds because of lemma 2. If all basic relations are serial, the condition for semi-associativity holds, because, by lemma $5,(a ; 1)=1$ for all basic relations $a$.

\subsection{What About Associativity?}

The non associative algebras we get are not in general associative. E.g., the algebra of Example 1 is not associative: $\left(\left(r_{1} ; r_{2}\right) ; r_{2}\right)=\left(\mathbf{1}^{\prime} ; r_{2}\right)=r_{2}$, whereas $\left(r_{1} ;\left(r_{2} ; r_{2}\right)\right)$ $=\left(r_{1} ; \mathbf{0}\right)=\mathbf{0}$. Although it satisfies the axiom of weak associativity [18], it is not semi-associative, since for instance $\left(r_{1} ; \mathbf{1}\right) ; \mathbf{1}=\mathbf{1}$ whereas $r_{1} ;(\mathbf{1} ; \mathbf{1})=r_{1}+\mathbf{1}^{\prime}$.

If weak composition coincides with composition, then the family $\left(R_{i}\right)_{i \in I}$ is a proper relation algebra, hence in particular it is associative. However, this sufficient condition is not necessary, as Example 2 shows: although the structure on the linear ordering on three elements has a weak composition which is not composition, it defines the point algebra, which is a relation algebra, hence associative. An example of an algebra which is semi-associative but not associative is the INDU calculus [6]. The semi-associativity of INDU is a consequence of the fact that all basic relations are serial.

\section{Weak Representations}

In the previous section, we showed how a qualitative calculus can be defined, starting from a partition scheme. The algebraic structure we get in this way is a non-associative algebra, i.e., an algebra that satisfies all axioms of a relation algebra, except possibly associativity.

Conversely, what is the nature of a partition scheme with respect to the algebra? The answer is that it is a weak representation of that algebra. The notion of a weak representation we use here ${ }^{3}$ was first introduced in [13] for relational algebras. It extends in a natural way to non-associative algebras.

Definition 3. Let $\mathcal{A}$ be a non-associative algebra. $A$ weak representation of $\mathcal{A}$ is a pair $(U, \varphi)$ where $U$ is a non empty set, and $\varphi$ is a map of $\mathcal{A}$ into $\mathcal{P}(U \times U)$, such that:

1. $\varphi$ is an homomorphism of Boolean algebras. 3. $\varphi\left(a^{\smile}\right)$ is the transpose of $\varphi(a)$. 2. $\varphi\left(\mathbf{1}^{\prime}\right)=\Delta=\{(x, y) \in U \times U \mid x=y\} . \quad$ 4. $\varphi(a ; b) \supseteq \varphi(a) \circ \varphi(b)$.

A weak representation is a representation if moreover:
5. $\varphi$ is injective.
6. $\varphi(a ; b)=\varphi(a) \circ \varphi(b)$.

Example 6. Take a set $U=\left\{u_{1}, u_{2}, u_{3}\right\}$ with three elements. Let $\varphi$ be defined by: $\varphi(\mathrm{o})=\left\{\left(u_{1}, u_{2}\right)\right\}, \varphi\left(\mathrm{o}^{-}\right)=\left\{\left(u_{2}, u_{1}\right)\right\}, \varphi(\mathrm{m})=\left\{\left(u_{1}, u_{3}\right)\right\}, \varphi\left(\mathrm{m}^{-}\right)=\left\{\left(u_{3}, u_{1}\right)\right\}$, $\varphi(\mathrm{d})=\left\{\left(u_{3}, u_{2}\right)\right\}, \varphi\left(\mathrm{d}^{\smile}\right)=\left\{\left(u_{2}, u_{3}\right)\right\}, \varphi(\mathrm{eq})=\left\{\left(u_{1}, u_{1}\right),\left(u_{2}, u_{2}\right),\left(u_{3}, u_{3}\right)\right\}$, and $\varphi(a)=\emptyset$ for any other basic relation $a$ in Allen's algebra. Then $(U, \varphi)$ is a weak representation of Allen's algebra which can be visualized as shown in Fig. 1(a).

\footnotetext{
${ }^{3}$ This notion is not to be confused with weak representability as used by Jónsson, see $[11,10]$.
} 
(a)

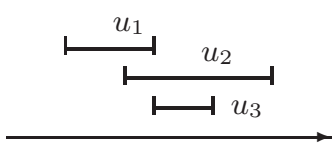

(b)

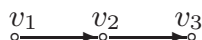

Fig. 1. A weak representation of Allen's algebra (a) and of the point algebra (b).

Example 7 (The point algebra). A weak representation of this algebra is a pair $(U, \prec)$, where $U$ is a set and $\prec$ is a linear ordering on $U$. It is a representation iff $\prec$ is dense and unbounded. Fig. 1(b) shows a weak representation with three points $v_{1}, v_{2}, v_{3}$.

\subsection{Partition Schemes and Weak Representations}

Now we come back to the original situation where we have a universe $U$ and a partition of $U \times U$ constituting a partition scheme. Consider the pair $(U, \varphi)$, where $\varphi: \mathcal{A} \rightarrow$ $\mathcal{P}(U \times U)$ is defined on the basic symbols by:

$$
\varphi\left(r_{i}\right)=R_{i}
$$

and is extended to the Boolean algebra in the natural way:

$$
\text { For } a \in \mathcal{A} \text { let } \varphi(a)=\bigcup_{r_{i} \in a} \varphi\left(r_{i}\right)
$$

Proposition 2. Given a partition scheme on $U$, define $\varphi$ as above. Then the pair $(U, \varphi)$ is a weak representation of $\mathcal{A}$.

Proof. The only point needing a proof is concerned with axiom 4 . For basic symbols, $\varphi\left(r_{i} ; r_{j}\right)=R_{i} \diamond R_{j}$, by definition, while $\varphi\left(r_{i}\right) \circ \varphi\left(r_{j}\right)=R_{i} \circ R_{j}$. By lemma 1 , the former relation contains the latter. The results extends to unions of relations.

From this proposition we can assert the (obvious) corollary:

Corollary 1. The weak representation associated to a partition scheme is a representation if and only if weak composition coincides with composition.

\section{What Is a Qualitative Calculus?}

We now have a general answer to our initial question: what is a qualitative calculus?

Definition 4. A qualitative calculus is a triple $(\mathcal{A}, U, \varphi)$ where:

1. $\mathcal{A}$ is a non-associative algebra.

2. $(U, \varphi)$ is a weak representation of $\mathcal{A}$. 


\subsection{The Ubiquity of Weak Representations}

Summing up, we started with a partition scheme and derived an algebra from it. This algebra, in all cases, is a non-associative algebra. It may or may not be a relation algebra. If the partition scheme is serial, it is a semi-associative algebra. In all cases, anyway, the original partition scheme defines a weak representation of the algebra.

In the next sections, we show that weak representations appear both as constraints (a-closed, normalized atomic networks) and as universes of interpretation. Consequently, many notions of consistency are related to morphisms between weak representations.

\subsection{Weak Representations as Constraint Networks}

Recall that a (finite) constraint network on $\mathcal{A}$ is a pair $\mathcal{N}=(N, \nu)$, where $N$ is a (finite) set of nodes (or variables) and $\nu$ a map $\nu: N \times N \rightarrow \mathcal{A}$. For each pair $(i, j)$ of nodes, $\nu(i, j)$ is the constraint on the $\operatorname{arc}(i, j)$. A network is atomic if $\nu$ is in fact a map into the set of basic relations (or atoms) of $\mathcal{A}$. It is normalized if $\forall i, j \in N \nu(i, j)=\mathbf{1}^{\prime}$ if $i=j$, and $\forall i, j \in N \nu(j, i)=\nu(i, j) \smile$. A network $\mathcal{N}^{\prime}=\left(N, \nu^{\prime}\right)$ is a refinement of $\mathcal{N}$ if $\forall i, j \in N$ we have $\nu^{\prime}(i, j) \subseteq \nu(i, j)$. Finally, a network is algebraically closed, or a-closed, if $\forall i, j, k \in N \nu(i, j) \subseteq \nu(i, k) ; \nu(k, j)$.

Let $(N, \nu)$ be a network, and consider for each atom $a \in \mathcal{A}$ the set $\rho(a)=\{(i, j) \in$ $N \times N \mid \nu(i, j)=a\}$. This defines a map from the set of atoms of $\mathcal{A}$ to the set of subsets of $N \times N$, which is interpreted as providing the set of arcs in the network which are labeled by a given atom. If the network is atomic, any arc is labeled by exactly one atom, i.e., the set of non-empty $\rho(a)$ is a partition of $N \times N$ labeled by atoms of $\mathcal{A}$. If it is normalized, this partition satisfies the conditions (2) and (3) characterizing a partition scheme. If the network is a-closed, then $(N, \rho)$, where $\rho$ is extended to $\mathcal{A}$ in the natural way, i.e., as $\rho(b)=\sum_{a \in b} \rho(a)$, is together with $N$ a weak representation of $\mathcal{A}$.

Conversely, for any weak representation $(U, \varphi)$, we can interpret $U$ as a set of nodes, and $\varphi\left(r_{i}\right)$ as the set of arcs labeled by $r_{i}$. Hence each arc is labeled by a basic relation, in such a way that $(v, u)$ is labeled by $r_{i}^{\smile}$ if $(u, v)$ is labeled by $r_{i}$, and that for all $u, v, w$ the composition of the label on $(u, w)$ with that on $(w, v)$ contains the label on $(u, v)$. Hence a weak representation is an a-closed, normalized atomic network.

Considering a weak representation in terms of a constraint network amounts to seeing it as an intensional entity: it expresses constraints on some instantiation of the variables of the network. Now, weak representations are at the same time extensional entities: as already apparent in the discussion of partition schemes, they also appear as universes of interpretation.

\subsection{Weak Representations as Interpretations}

Many standard interpretations of qualitative calculi are particular kinds of weak representations of the algebra, namely, representations. Allen's calculus, e.g., is usually interpreted in terms of the representation provided by "intervals", in the sense of strictly increasing pairs in the rational or real line. It has less been pointed out in the literature that in many cases weak representations, rather than representations, are what the calculi are actually about. 


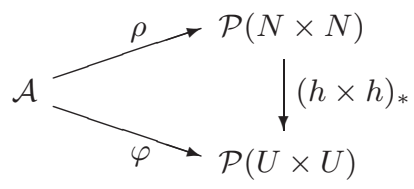

Fig. 2. A general notion of consistency.

As already discussed in [13], a finite weak representation of Allen's algebra can be visualized in terms of finite sets of intervals on a finite linear ordering. More generally, restricting the calculus to some sub-universe amounts to considering weak representations of Allen's algebra: for instance, considering intervals on the integers (Example 5) yields a weak representation. It also makes sense to consider the problem of determining whether constraint networks are consistent with respect to this restrictive interpretation.

Encountering the notion of seriality is not surprising. Recall that a constraint network is $k$-consistent if any instantiation of $k-1$ variables extends to $k$-variables. In particular, a network is 2-consistent if any instantiation of one variable extends to two variables. Hence a partition scheme is serial if and only if the (possibly infinite) "network" $U$ (or weak representation) is 2-consistent. Many natural calculi have consistent networks which are not 2-consistent, e.g., Allen's calculus on integers. Although the 2 -element network with constraint $\mathrm{d}$ is consistent, it is not 2-consistent: if an interval $x$ has length one, there is no interval $y$ such that $y \mathrm{~d} x$.

\section{What Is Consistency?}

The preceding discussion shows that a weak representation can be considered alternatively as a particular kind of constraint network (an atomic, normalized and a-closed one), or as a universe of interpretation. Now, a fundamental question about a network is whether it is consistent with respect to a given domain of interpretation.

Intuitively, a network $\mathcal{N}=(N, \nu)$ is consistent (with respect to a calculus $(\mathcal{A}, U, \varphi))$ if it has an atomic refinement $\mathcal{N}^{\prime}=\left(N, \nu^{\prime}\right)$ which is itself consistent, that is, the variables $N$ of $\mathcal{N}$ can be interpreted in terms of elements of $U$ in such a way that the relations prescribed by $\nu^{\prime}$ hold in $U$. More specifically, if $\left(N, \nu^{\prime}\right)$ is a-closed, normalized, and atomic, consider the associated weak representation $(N, \rho)$. Then the consistency of the network with respect to the weak representation $(U, \varphi)$ means that there exists an instantiation $h: N \rightarrow U$ such that, for each atom $a \in \mathcal{A},(i, j) \in \rho(a)$ implies $(h(i), h(j)) \in \varphi(a)$. Hence consistency of such a network appears as a particular case of compatibility between two weak representations. This means that in fact consistency is a property involving two weak representations:

Definition 5. Let $\mathcal{N}=(N, \rho)$ and $\mathcal{U}=(U, \varphi)$ be two weak representations of $\mathcal{A}$. Then $\mathcal{N}$ is consistent with respect to $\mathcal{U}$ if there exists a map $h: N \rightarrow U$ such that the diagram in Fig. 2 commutes, that is, for each $a \in \mathcal{A},(i, j) \in \rho(a)$ implies $(h(i), h(j)) \in \varphi(a)$.

This generalization of the notion of consistency emphasizes the fact that it is a notion between two weak representations, where one is interpreted in intentional terms, while the other is used in an extensional way, as a universe of interpretation. 
Example 8 (The point algebra). A weak representation in that case is a linearly ordered set. Consider two such weak representations $\left(N, \prec_{N}\right)$ and $\left(U, \prec_{U}\right)$. Then $\left(N, \prec_{N}\right)$ is consistent with respect to $\left(U, \prec_{U}\right)$ iff there is a strictly increasing map $h: N \rightarrow U$.

\subsection{Inconsistent Weak Representations}

In that light, what is the meaning of the existence of inconsistent weak representations? Examples of finite atomic a-closed networks which are not consistent exist e.g. for the cyclic interval calculus or the INDU calculus [17]. In such cases, the universe of interpretation of the calculus (such as intervals on a rational circle, or intervals with duration) has too much additional structure and constraints on its relations for the network to take them into account. Characterizing the cases where this can happen seems to be an open problem in general.

\section{Conclusions}

This paper proposes to introduce a shift of perspective in the way qualitative calculi are considered. Since Allen's calculus has been considered as a paradigmatic instance of a qualitative calculus for more than two decades, it has been assumed that the algebraic structures governing them are relation algebras, and that the domains of interpretation of the calculi should in general be extensional or, in algebraic terms, representations of these algebras. These assumptions, however, have been challenged by a series of facts: some calculi, as first shown in [9], then by [17], involve non-associative algebras. Also, for many calculi, the domains of interpretation may vary, and do not necessarily constitute representations.

We argued in this paper that a qualitative calculus should be defined abstractly as a triple consisting of a non-associative algebra and a weak representation of that algebra. This abstract definition makes apparent the fact that particular kinds of networks on the one side, and representations of the algebras on the other side, are ultimately of a common nature, namely, both are particular kinds of weak representations. This last fact has of course been known before: for instance, the work described in [10] is about trying to construct representations of a given relation algebra by incrementally enriching a-closed networks using games à la Ehrenfeucht-Fraissé. However, we think that putting qualitative calculi in this setting provides a clear way of considering new calculi, as well as an agenda for questions to be asked first: what are the properties of the algebra involved? What are weak representations? Are the intended interpretations representations of the algebra? When are weak representations consistent with respect to which weak representations?

A further benefit of the framework is that it makes clearly apparent what consistency really means: consistency of a network (a network is a purely algebraic notion) with respect to the calculus is a particular case of consistency between two weak representations: it can be defined as the possibility of refining the network into a weak representation which is consistent wrt. the one which is part of the calculus considered.

Obviously, defining a general framework is only an initial step for studying the new problems which arise for calculi which are less well-behaved than Allen's calculus. 
A first direction of investigation we are currently exploring consists in trying to get a better understanding of the relationship between consistency and the expressiveness of constraint networks.

\section{References}

1. J. F. Allen. Maintaining knowledge about temporal intervals. CACM, 26(11):832-843, 1983.

2. P. Balbiani and A. Osmani. A model for reasoning about topologic relations between cyclic intervals. In Proc. of KR-2000, Breckenridge, Colorado, 2000.

3. P. Balbiani, J.-F. Condotta, and L. Fariñas del Cerro. A model for reasoning about bidimensional temporal relations. In Proc. of KR-98, p. 124-130, 1998.

4. P. Balbiani, J.-F. Condotta, and L. Fariñas del Cerro. Spatial reasoning about points in a multidimensional setting. In Proc. IJCAI'99 Spat.\&Temp. Reasoning WS, p.105-113, 1999.

5. P. Balbiani, J.-F. Condotta, and L. Fariñas del Cerro. A tractable subclass of the block algebra: constraint propagation and preconvex relations. In Proc. EPIA'99, p. 75-89, 1999.

6. P. Balbiani, J.-F. Condotta, and G. Ligozat. On the Consistency Problem for the INDU Calculus. In Proc. TIME-ICTL-2003, Cairns, Australia, 2003.

7. R. Dechter, I. Meiri, and J. Pearl. Temporal Constraint Networks. AIJ, 49(1-3):61-95, 1991.

8. I. Duentsch and M. Roubens. Tangent circle algebras. In H. de Zwart, editor, Relational Methods in Computer Science, p. 300-314. LNCS, Vol.2561. Springer, 2002.

9. M. Egenhofer and A. Rodriguez. Relation Algebras over Containers and Surfaces: An Ontological Study of a Room Space. Spatial Cognition and Computation, 1(2):155-180, 1999.

10. R. Hirsch and I. Hodkinson. Relation Algebras by Games. North Holland, 2002.

11. B. Jónsson. Representation of modular lattices and relation algebras. Tr. AMS, 92:449-464, 1959.

12. P. Ladkin and R. Maddux. On Binary Constraint Problems. JACM, 41(3):435-469, 1994.

13. G. Ligozat. Weak Representations of Interval Algebras. In Proc. AAAI'90, p. 715-720, 1990.

14. G. Ligozat. On generalized interval calculi. In Proc. AAAI'91, p. 234-240, 1991.

15. G. Ligozat. Tractable relations in temporal reasoning: pre-convex relations. In Proc. of the ECAI-94 Workshop on Spatial and Temporal Reasoning, p. 99-108, Amsterdam, 1994.

16. G. Ligozat. Reasoning about cardinal directions. J. of Vis. Lang. \& Comp., 1(9):23-44, 1998.

17. G. Ligozat, D. Mitra, and J.-F. Condotta. Spatial and Temporal Reasoning: Beyond Allen's Calculus. In Proc. AAAI Symp. on Found. and Appl. of Spatio-Temporal Reasoning, 2003.

18. R. Maddux. Some varieties containing relation algebras. Trans. AMS, 272(2):501-526, 1982.

19. D. Mitra. Qualitative Reasoning with Arbitrary Angular Directions. In Proc. of the AAAI-02 W20 Workshop on Spatial and Temporal Reasoning, Edmonton, Canada, 2002.

20. A. K. Pujari and A. Sattar. A new framework for reasoning about points, intervals and durations. In Proc. IJCAI'99), p. 1259-1267, 1999.

21. D. A. Randell, Z. Cui and A. G. Cohn. A spatial logic based on regions and connection. In Proc. KR'92, p. 165-176, 1992.

22. J. Renz. A spatial Odyssey of the interval algebra: 1. Directed intervals. In Proc. IJCAI'01, p.51-56, 2001.

23. A. Tarski. On the calculus of relations. Journal of Symbolic Logic, 6:73-89, 1941.

24. M. B. Vilain and H. Kautz. Constraint propagation algorithms for temporal reasoning. In Proc. of AAAI-86, p. 377-382, 1986. 